\title{
Models of Non-Minimal Chaotic Inflation in Supergravity
}

\section{Pallis*}

Department of Physics, University of Cyprus, P.O. Box 20537, Nicosia 1678, CYPRUS

E-mail: cpalliseucy.ac.cy

\begin{abstract}
We show how we can implement chaotic inflation in the context of supergravity by conveniently selecting the functional form of a strong enough non-minimal coupling between the inflaton and the Ricci scalar curvature. The procedure can be applied when a gauge singlet or non-singlet inflaton is coupled to another singlet superfield within linear-quadratic, trilinear or bilinear superpotential terms. The tachyonic instability occurring along the direction of the accompanying non-inflaton field can be cured by expanding the kinetic part of the frame function up to the fourth order in powers of the various fields. In the case of a gauge non-singlet inflaton, though, a conjugation symmetry has to be imposed on these terms in order for the flatness of the inflationary potential is maintained. On the other hand, some of these terms assist us to precisely reconcile the resulting scalar spectral index with the current PLANCK measurements while the other inflationary observables are in agreement with data.
\end{abstract}

Proceedings of the Corfu Summer Institute 2012

September 8-27, 2012

Corfu, Greece

\footnotetext{
* Speaker.
} 


\section{Introduction}

Non-minimal (chaotic) inflation ( $\mathrm{nMl}$ ) is a class of inflationary models which arises in the presence of a strong enough non-minimal coupling function between the inflaton field and the Ricci scalar curvature. In this talk, which is based on Refs. [1-4], we first briefly review the basic ingredients of nMI in a non-Supersymmetric (SUSY) framework (Sec. II) and constrain the parameters of two typical models in Sec. [.3 taking into account the observational requirements described in Sec. [2. Throughout the text, the subscript, $\chi$ denotes derivation with respect to (w.r.t) the field $\chi$ and we follow the conventions of Ref. [1].

\subsection{Coupling non-Minimally the Inflaton to Gravity}

The action of an inflaton $\phi$ with potential $V_{\mathrm{I}}(\phi)$ non-minimally coupled to Ricci scalar $R$ through a coupling function $f_{R}(\phi)$, in the Jordan frame (JF), takes the form:

$$
\mathrm{S}=\int d^{4} x \sqrt{-\mathfrak{g}}\left(-\frac{1}{2} m_{\mathrm{P}}^{2} f_{R}(\phi) R+\frac{f_{K}(\phi)}{2} g^{\mu v} \partial_{\mu} \phi \partial_{\nu} \phi-V_{\mathrm{I}}(\phi)\right)
$$

where $m_{\mathrm{P}}=2.44 \cdot 10^{18} \mathrm{GeV}$ is the reduced Planck mass, $\mathfrak{g}$ is the determinant of the background Friedmann-Robertson-Walker metric, $g^{\mu \nu}$. We allow also for a kinetic mixing through the function $f_{K}(\phi)$. We can write $\mathrm{S}$ in the Einstein frame (EF) as follows

$$
\mathrm{S}=\int d^{4} x \sqrt{-\widehat{\mathfrak{g}}}\left(-\frac{1}{2} m_{\mathrm{P}}^{2} \widehat{R}+\frac{1}{2} \widehat{g}^{\mu v} \partial_{\mu} \widehat{\phi} \partial_{\nu} \widehat{\phi}-\widehat{V}_{\mathrm{I}}(\widehat{\phi})\right)
$$

by performing a conformal transformation [1] according to which we define the EF metric

$$
\widehat{g}_{\mu v}=f_{R} g_{\mu v} \Rightarrow\left\{\begin{array}{l}
\sqrt{-\widehat{\mathfrak{g}}}=f_{R}^{2} \sqrt{-\mathfrak{g}} \text { and } \widehat{g}^{\mu v}=g^{\mu v} / f_{R}, \\
\widehat{R}=\left(R+3 \square \ln f_{R}+3 g^{\mu v} \partial_{\mu} f_{R} \partial_{v} f_{R} / 2 f_{R}^{2}\right) / f_{R},
\end{array}\right.
$$

where $\square=(-\mathfrak{g})^{-1 / 2} \partial_{\mu}\left(\sqrt{-\mathfrak{g}} \partial^{\mu}\right)$ and hat is used to denote quantities defined in the EF. We also introduce the EF canonically normalized field, $\widehat{\phi}$, and potential, $\widehat{V}_{\mathrm{I}}$, defined as follows:

$$
\left(\frac{d \widehat{\phi}}{d \phi}\right)^{2}=J^{2}=\frac{f_{K}}{f_{R}}+\frac{3}{2} m_{\mathrm{P}}^{2}\left(\frac{f_{R, \phi}}{f_{R}}\right)^{2} \text { and } \widehat{V}(\widehat{\phi})=\frac{V_{\mathrm{I}}(\widehat{\phi}(\phi))}{f_{R}(\widehat{\phi}(\phi))^{2}} .
$$

For convenient choices of $V_{\mathrm{I}}(\phi)$ and $f_{R}(\phi)$ we can obtain a sufficiently flat $\widehat{V}_{\mathrm{I}}(\widehat{\phi})$ which can support nMI. The analysis of nMI in the EF using the standard slow-roll approximation is equivalent with the analysis in JF. We have just to keep in mind the dependence of $\widehat{\phi}$ on $\phi$.

\subsection{Inflationary Observables - Constraints}

Under the assumptions that (i) the curvature perturbation generated by $\phi$ is solely responsible for the observed one and (ii) nMI is followed in turn by a decaying-inflaton, radiation and matter domination, the parameters of nMI can be restricted by requiring that: 


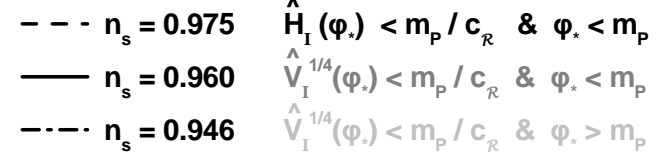

Figure 1: Conventions for the type and the color of the lines used for the various restrictions on the parameters of our models.

(i) The number of e-foldings, $\widehat{N}_{*}$, that the scale $k_{*}=0.05 / \mathrm{Mpc}$ suffers during nMI, leads to a solution of the horizon and flatness problems of standard big bang, i.e., [5]

$$
\int_{\widehat{\phi}_{\mathrm{f}}}^{\widehat{\phi}_{*}} \frac{d \widehat{\phi}}{m_{\mathrm{P}}^{2}} \frac{\widehat{V}_{\mathrm{I}}}{\widehat{V}_{\mathrm{I}, \widehat{\phi}}}=\int_{\phi_{\mathrm{f}}}^{\phi_{*}} J^{2} \frac{\widehat{V}_{\mathrm{I}}}{\widehat{V}_{\mathrm{I}, \phi}} \frac{d \phi}{m_{\mathrm{P}}^{2}} \simeq 19.4+2 \ln \frac{V_{\mathrm{I}}\left(\phi_{*}\right)^{1 / 4}}{1 \mathrm{GeV}}-\frac{4}{3} \ln \frac{V_{\mathrm{I}}\left(\phi_{\mathrm{f}}\right)^{1 / 4}}{1 \mathrm{GeV}}+\frac{1}{3} \ln \frac{T_{\mathrm{rh}}}{1 \mathrm{GeV}}+\frac{1}{2} \ln \frac{f_{R}\left(\phi_{\mathrm{f}}\right)}{f_{R}\left(\phi_{*}\right)},
$$

where $\phi_{\mathrm{f}}\left[\widehat{\phi}_{\mathrm{f}}\right]$ is the value of $\phi[\widehat{\phi}]$ at the end of nMI, which can be found, in the slow-roll approximation and for the considered in this paper models, from the condition

$$
\begin{gathered}
\max \left\{\widehat{\varepsilon}\left(\phi_{\mathrm{f}}\right),\left|\widehat{\eta}\left(\phi_{\mathrm{f}}\right)\right|\right\}=1 \text {, where } \\
\widehat{\varepsilon}=\frac{m_{\mathrm{P}}^{2}}{2}\left(\frac{\widehat{V}_{\mathrm{I}, \widehat{\phi}}}{\widehat{V}_{\mathrm{I}}}\right)^{2}=\frac{m_{\mathrm{P}}^{2}}{2 J^{2}}\left(\frac{\widehat{V}_{\mathrm{I}, \phi}}{\widehat{V}_{\mathrm{I}}}\right)^{2} \text { and } \widehat{\eta}=m_{\mathrm{P}}^{2} \frac{\widehat{V}_{\mathrm{I}, \widehat{\phi} \widehat{\phi}}}{\widehat{V}_{\mathrm{I}}}=\frac{m_{\mathrm{P}}^{2}}{J^{2}}\left(\frac{\widehat{V}_{\mathrm{I}, \phi \phi}}{\widehat{V}_{\mathrm{I}}}-\frac{\widehat{V}_{\mathrm{I}, \phi}}{\widehat{V}_{\mathrm{I}}} \frac{J_{, \phi}}{J}\right) .
\end{gathered}
$$

Also $T_{\mathrm{rh}}$ is the reheat temperature after nMI, which is taken $T_{\mathrm{rh}}=10^{8} \mathrm{GeV}$ throughout, since its variation over some orders of magnitude influences only weakly $\widehat{N}_{*}$, which remains close to 50 .

(ii) The amplitude $A_{\mathrm{s}}$ of the power spectrum of the curvature perturbation generated by $\phi$ at the pivot scale $k_{*}$ is consistent with data [5]

$$
\sqrt{A_{\mathrm{S}}}=\frac{1}{2 \sqrt{3} \pi m_{\mathrm{P}}^{3}} \frac{\widehat{V}_{\mathrm{I}}\left(\widehat{\phi}_{*}\right)^{3 / 2}}{\left|\widehat{V}_{\mathrm{I}, \hat{\phi}}\left(\widehat{\phi}_{*}\right)\right|}=\frac{\left|J\left(\phi_{*}\right)\right|}{2 \sqrt{3} \pi m_{\mathrm{P}}^{3}} \frac{\widehat{V}_{\mathrm{I}}\left(\phi_{*}\right)^{3 / 2}}{\left|\widehat{V}_{\mathrm{I}, \phi}\left(\phi_{*}\right)\right|} \simeq 4.685 \cdot 10^{-5}
$$

where $\phi_{*}\left[\widehat{\phi}_{*}\right]$ is the value of $\phi[\widehat{\phi}]$ when $k_{*}$ crosses outside the inflationary horizon.

(iii) The (scalar) spectral index, $n_{\mathrm{s}}$, its running, $a_{\mathrm{s}}$, and the scalar-to-tensor ratio $r$ - estimated through the relations:

$$
n_{\mathrm{s}}=1-6 \widehat{\mathcal{\varepsilon}}_{*}+2 \widehat{\eta}_{*}, \quad \alpha_{\mathrm{s}}=2\left(4 \widehat{\eta}_{*}^{2}-\left(n_{\mathrm{s}}-1\right)^{2}\right) / 3-2 \widehat{\xi}_{*} \text { and } r=16 \widehat{\varepsilon},
$$

where $\widehat{\xi}=m_{\mathrm{P}}^{4} \widehat{V}_{\mathrm{I}, \widehat{\phi}} \widehat{V}_{\mathrm{I}, \hat{\phi} \hat{\phi} \hat{\phi}} / \widehat{V}^{2}=m_{\mathrm{P}}^{2} \widehat{V}_{\mathrm{I}, \phi} \widehat{\eta}_{, \phi} / \widehat{V}_{\mathrm{I}} J^{2}+2 \widehat{\eta} \widehat{\varepsilon}$ and the variables with subscript $*$ are evaluated at $\phi=\phi_{*}-$ are consistent with the fitting of the data [5] with $\Lambda \mathrm{CDM}$ model, i.e.,

$$
\text { (a) } n_{\mathrm{s}}=0.9603 \pm 0.0146, \text { (b) }-0.0314 \leq a_{\mathrm{s}} \leq 0.0046 \text { and (c) } r<0.11 \text {, }
$$

at $95 \%$ confidence level (c.1.)

(iv) The effective theory describing nMI remains valid. This aim can be achieved if the ultraviolet cut-off scale [6], $\Lambda_{\text {eff }}$, remains larger than the inflationary scale which is represented by $\widehat{V}_{\mathrm{I}}\left(\phi_{*}\right)^{1 / 4}$ or, less restrictively, by the corresponding Hubble parameter, $\widehat{H}_{\mathrm{I} *}=\widehat{V}_{\mathrm{I}}\left(\phi_{*}\right)^{1 / 2} / \sqrt{3} m_{\mathrm{P}}$, i.e.

$$
\text { (a) } \widehat{V}_{\mathrm{I}}\left(\phi_{*}\right)^{1 / 4} \leq \Lambda_{\text {eff }} \text { or (b) } \widehat{H}_{\mathrm{I} *} \leq \Lambda_{\text {eff }} \text { with } \Lambda_{\text {eff }} \simeq m_{\mathrm{P}} / c_{R} \text {. }
$$

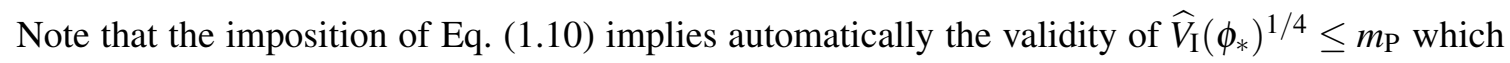
is necessary in order to avoid possible corrections from quantum gravity. Less favored, from theoretical point of view, is also the domain of parameters where $\phi_{*} \geq m_{\mathrm{P}}$. The conventions adopted for the description of the various restrictions on the parameters of our models are shown in Fig. $\square$. 


\subsection{Non-SUSY Models of nMI}

Using the criteria of Sec. $\mathbb{L}$, it would be instructive to test two simple models of inflation

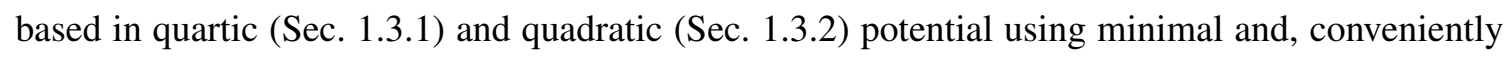
chosen, non-minimal coupling functions.

\subsubsection{The Quartic Potential}

Adopting the potential $V_{\mathrm{I}}=\lambda \phi^{4} / 4$ for the inflaton $\phi$ we can analyze the cases:

(i) If we use $f_{R}=1$ and $f_{K}=1$, i.e. the minimal coupling to gravity, the slow-roll parameters and the number of $e$-foldings, suffered from $k_{*}$ during minimal inflation (MI) can be calculated applying Eq. (ㅁ.6) and Eq. (ㄷ.5) - after removing hats and setting $J=1$ - with results

$$
\varepsilon \simeq 8 m_{\mathrm{P}}^{2} / \phi^{2}, \quad \eta \simeq 12 m_{\mathrm{P}}^{2} / \phi^{2} \text { and } N_{*} \simeq \phi_{*}^{2} / 8 m_{\mathrm{P}}^{2}
$$

Therefore, the values of $\phi$ at the end of MI and the horizon crossing of $k_{*}$ are transplanckian, since

$$
\phi_{\mathrm{f}}=2 \sqrt{3} m_{\mathrm{P}} \text { and } \phi_{*}=2 \sqrt{2 N_{*}} m_{\mathrm{P}} .
$$

Moreover, the normalization of Eq. ([L]) imposes the condition

$$
\sqrt{A_{\mathrm{s}}} \simeq \frac{\sqrt{\lambda} \phi_{*}^{3}}{16 \sqrt{3} \pi m_{\mathrm{P}}^{3}}=4.685 \cdot 10^{-5} \Rightarrow \lambda^{1 / 2} \simeq \sqrt{\frac{3}{2}} 4.685 \cdot 10^{-5} \pi N_{*}^{-3 / 2} \Rightarrow \lambda \simeq 2 \cdot 10^{-13}
$$

for $N_{*} \simeq 53$. This value of $\lambda$ signals an ugly tuning. From Eq. (ㄷ.8) we get

$$
n_{\mathrm{s}} \simeq 1-3 / N_{*} \simeq 0.947, \alpha_{\mathrm{s}} \simeq-3 / N_{*}^{2} \simeq 9.5 \cdot 10^{-4} \text { and } r \simeq 16 / N_{*} \simeq 0.28,
$$

with the last value being in clear contradiction with Eq. ([L.9c).

(ii) If we employ $f_{R}(\phi)=1+c_{R}\left(\phi / m_{\mathrm{P}}\right)^{2}$ and $f_{K}=1$, i.e., the standard non-minimal coupling to gravity, we find from Eq. (ㄴ.4), for $c_{R} \gg 1$

$$
J \simeq \sqrt{6} m_{\mathrm{P}} / \phi \text { and } \widehat{V}_{\mathrm{I}}=\lambda \phi^{4} / 4 f_{R}^{2} \simeq \lambda m_{\mathrm{P}}^{4} / 4 c_{R}^{2} .
$$

We observe that $\widehat{V}_{\mathrm{I}}$ exhibits an almost flat plateau. From Eqs. (‥5) and (‥6) we find

$$
\widehat{\varepsilon} \simeq 4 m_{\mathrm{P}}^{4} / 3 c_{R}^{2} \phi^{4}, \widehat{\eta} \simeq-4 m_{\mathrm{P}}^{2} / 3 c_{R} \phi^{2} \text { and } \widehat{N}_{*} \simeq 3 c_{R} \phi_{*}^{2} / 4 m_{\mathrm{P}}^{2} .
$$

Therefore, $\phi_{\mathrm{f}}$ and $\phi_{*}$ are found from the condition of Eq. (L.6) and the last equality above, as follows

$$
\phi_{\mathrm{f}}=\sqrt[4]{4 / 3} m_{\mathrm{P}} / \sqrt{c_{R}} \text { and } \phi_{*}=2 m_{\mathrm{P}} \sqrt{\widehat{N}_{*} / 3 c_{R}}
$$

Also the normalization of Eq. ([L.]) implies the following relation between $c_{R}$ and $\sqrt{\lambda}$

$$
\sqrt{A_{\mathrm{s}}} \simeq \frac{\sqrt{\lambda} \widehat{N}_{*}}{6 \sqrt{2} \pi c_{R}}=4.685 \cdot 10^{-5} \Rightarrow c_{R} \simeq 4.2 \cdot 10^{4} \sqrt{\lambda}
$$

for $\widehat{N}_{*} \simeq 52$. Obviously $\lambda$ can be larger than this in Eq. (미). From Eq. (따) we get

$$
n_{\mathrm{s}} \simeq 1-2 / \widehat{N}_{*} \simeq 0.965, \alpha_{\mathrm{s}} \simeq-2 / \widehat{N}_{*}^{2} \simeq-6.4 \cdot 10^{-4} \text { and } r \simeq 12 / \widehat{N}_{*}^{2} \simeq 4 \cdot 10^{-3},
$$

which are in agreement with Eq. (매). If, in addition, we impose the requirement $\phi \leq m_{\mathrm{P}}$ and that of Eq. (미a) we end-up with following ranges - c.f. Ref. [1]:

$$
74 \simeq 4 \widehat{N} / 3 \lesssim c_{R} \lesssim 300 \text { and } 0.3 \lesssim \lambda / 10^{-5} \lesssim 4.6
$$

Therefore, the presence of $f_{R}$ can rescue the model based on the simplest quartic potential. 


\subsubsection{The Quadratic Potential}

Focusing on the potential $V_{\mathrm{I}}=m^{2} \phi^{2} / 2$ for the inflaton $\phi$ we concentrate on the cases:

(i) If we use $f_{R}=1$ and $f_{K}=1$, i.e. the minimal coupling to gravity, and work along the lines of

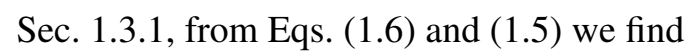

$$
\varepsilon=\eta=2 m_{\mathrm{P}}^{2} / \phi^{2} \text { and } N_{*}=\left(\phi_{*}^{2}-\phi_{\mathrm{f}}^{2}\right) / 4 m_{\mathrm{P}}^{2} .
$$

Imposing the condition of Eq. (ㅁ.6) and solving the second expression above w.r.t $\phi_{*}$ we find

$$
\phi_{\mathrm{f}}=\sqrt{2} m_{\mathrm{P}} \text { and } \phi_{*} \simeq 2 m_{\mathrm{P}} \sqrt{N_{*}} .
$$

As in Sec. ‥3. transplanckian $\phi$ 's are required. Enforcing Eq. (․ㄱ), we extract

$$
\sqrt{A_{\mathrm{s}}} \simeq \frac{m \phi_{*}^{2}}{4 \sqrt{6} \pi m_{\mathrm{P}}^{3}}=4.686 \cdot 10^{-5} \Rightarrow m \simeq 4.686 \cdot 10^{-5} \sqrt{6} \pi m_{\mathrm{P}} N_{*}^{-1} \Rightarrow m \simeq 10^{13} \mathrm{GeV},
$$

which can be related to the masses of the right-handed neutrinos [2]. Applying Eq. ([L.8) we get

$$
n_{\mathrm{s}} \simeq 1-2 / N_{*} \simeq 0.965, \quad \alpha_{\mathrm{s}} \simeq-2 / N_{*}^{2} \simeq-6.5 \cdot 10^{-4} \text { and } r \simeq 8 / N_{*} \simeq 0.13 .
$$

Although $n_{\mathrm{S}}$ is close to the observationally favored one, $r$ is in tension with Eq. (प्पc).

(ii) If we employ $f_{R}(\phi)=1+c_{R} \phi / m_{\mathrm{P}}$ and $f_{K}=1$, i.e. a linear non-minimal coupling to gravity, we find from Eq. (ㄴ. $)$, for $c_{R} \gg 1$

$$
J \simeq \sqrt{3 / 2} m_{\mathrm{P}} \phi^{-1} \text { and } \widehat{V}_{\mathrm{I}} \simeq m^{2} m_{\mathrm{P}}^{2} / 2 c_{R}^{2},
$$

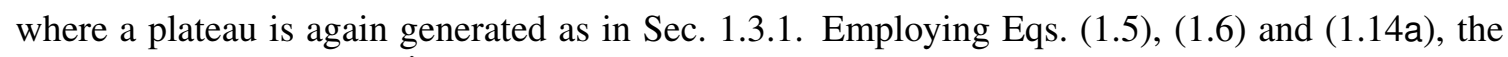
slow roll parameters and $\widehat{N}_{*}$ read

$$
\widehat{\varepsilon} \simeq 4 m_{\mathrm{P}} / 3 c_{R}^{2} \phi^{2}, \hat{\eta} \simeq-4 m_{\mathrm{P}} / 3 c_{R} \phi \text { and } \widehat{N}_{*} \simeq 3 c_{R} \phi_{*} / 4 m_{\mathrm{P}}
$$

Imposing the condition of Eq. (ㄸ.6) and solving then the latter equation w.r.t $\phi_{*}$ we arrive at

$$
\phi_{\mathrm{f}} \simeq 2 m_{\mathrm{P}} / \sqrt{3} c_{R} \text { and } \phi_{*} \simeq 4 m_{\mathrm{P}} \widehat{N}_{*} / 3 c_{R} .
$$

On the other hand, Eq. (피) implies a relation between $m$ and $c_{R}-$ cf. Eq. (ㄷ.J2d)

$$
\sqrt{A_{\mathrm{s}}} \simeq \frac{m \widehat{N}_{*}}{6 \pi m_{\mathrm{P}} c_{R}}=4.686 \cdot 10^{-5} \Rightarrow m=4.13 \cdot 10^{13} c_{R} \mathrm{GeV} .
$$

Applying Eq. ([.8) we find that the predictions of the model w.r.t $n_{\mathrm{s}}, \alpha_{\mathrm{s}}$ and $r$ are identical to those in Eq. ([L.2E). If, in addition, we impose the requirement $\phi \leq m_{\mathrm{P}}$ and that of Eq. (다la) we end-up with following ranges - c.f. Ref. [1]:

$$
77 \simeq 4 \widehat{N}_{*} / 3 \lesssim c_{R} \lesssim 313 \text { and } 2.9 \lesssim m / 10^{15} \mathrm{GeV} \lesssim 12,
$$

where the ranges above are derived numerically. Therefore, the presence of a linear $f_{R}$ renders observationally more interesting the model based on the simplest quadratic potential. 


\subsection{Outline}

It would be certainly interesting to enquire if it is possible to realize similar models of nMI in a SUSY framework where the hierarchy problem of Grand Unified Theories (GUTs) is elegantly resolved. We below describe the formulation of nMI in the context of Supergravity (SUGRA) and we specify three models of nMI: two with a gauge singlet inflaton (coupled to another gauge singlet in a linear-quadratic or a bilinear superpotential term) and one with a gauge non-singlet inflaton.

\section{Realization of nMI Within SUGRA}

In Sec. De present the basic formulation of a theory which exhibits non-minimal coupling of scalar fields to $R$ within SUGRA and in Sec. 2.2 we outline our strategy in constructing viable models of nMI.

\subsection{The General Set-up}

Our starting point is the EF action for the scalar fields $\Phi^{\alpha}$ within SUGRA $[2,7]$ which can be written as

$$
\mathrm{S}=\int d^{4} x \sqrt{-\widehat{\mathfrak{g}}}\left(-\frac{1}{2} m_{\mathrm{P}}^{2} \widehat{R}+K_{\alpha \bar{\beta}} \widehat{g}^{\mu v} D_{\mu} \Phi^{\alpha} D_{v} \Phi^{* \bar{\beta}}-\widehat{V}\right)
$$

where the following notation is adopted

$$
K_{\alpha \bar{\beta}}=K_{, \Phi^{\alpha} \Phi^{* \bar{\beta}}}>0 \text { and } D_{\mu} \Phi^{\alpha}=\partial_{\mu} \Phi^{\alpha}-A_{\mu}^{A} k_{A}^{\alpha}
$$

are the covariant derivatives for scalar fields $\Phi^{\alpha}$. Here and henceforth the scalar components of the various superfields are denoted by the same superfield symbol. Also $A_{\mu}^{A}$ stand for the vector gauge fields and $k_{A}^{\alpha}$ is the Killing vector, defining the gauge transformations of the scalars [7]. The EF potential, $\widehat{V}$, is given in terms of the Kähler potential, $K$, and the superpotential, $W$, by

$$
\widehat{V}=\widehat{V}_{\mathrm{F}}+\widehat{V}_{\mathrm{D}} \text { with } \widehat{V}_{\mathrm{F}}=e^{K / m_{\mathrm{P}}^{2}}\left(K^{\alpha \bar{\beta}} \mathrm{F}_{\alpha} \mathrm{F}_{\bar{\beta}}^{*}-3 \frac{|W|^{2}}{m_{\mathrm{P}}^{2}}\right) \text { and } \widehat{V}_{\mathrm{D}}=\frac{1}{2} g^{2} \sum_{a} \mathrm{D}_{a} \mathrm{D}_{a} .
$$

Here, $g$ is the unified gauge coupling constant and the summation is applied over the generators $T_{a}$ of a considered gauge group - a trivial gauge kinetic function is adopted. Also we use the shorthand

$$
K^{\bar{\beta} \alpha} K_{\alpha \bar{\gamma}}=\delta_{\bar{\gamma}}^{\bar{\beta}}, \mathrm{F}_{\alpha}=W_{, \Phi^{\alpha}}+K_{, \Phi^{\alpha}} W / m_{\mathrm{P}}^{2} \text { and } \mathrm{D}_{a}=\Phi_{\alpha}\left(T_{a}\right)_{\beta}^{\alpha} K^{\beta} \text { with } K^{\alpha}=K_{, \Phi^{\alpha}} .
$$

By performing a conformal transformation and adopting a frame function $\Omega$ which is related to $K$ as follows

$$
-\Omega / 3=e^{-K / 3 m_{\mathrm{P}}^{2}} \Rightarrow K=-3 m_{\mathrm{P}}^{2} \ln (-\Omega / 3),
$$

we arrive at the following action

$$
\mathrm{S}=\int d^{4} x \sqrt{-\mathfrak{g}}\left(-\frac{m_{\mathrm{P}}^{2}}{2}\left(-\frac{\Omega}{3}\right) R+m_{\mathrm{P}}^{2} \Omega_{\alpha \bar{\beta}} D_{\mu} \Phi^{\alpha} D^{\mu} \Phi^{* \bar{\beta}}-\Omega \mathscr{A}_{\mu} \mathscr{A}^{\mu} / m_{\mathrm{P}}^{2}-V\right)
$$

where $g_{\mu v}=-(3 / \Omega) \widehat{g}_{\mu \nu}$ is the JF metric, we use the shorthand notation $\Omega_{\alpha}=\Omega_{, \Phi^{\alpha}}$ and $\Omega_{\bar{\alpha}}=$ $\Omega_{, \Phi^{* \bar{\alpha}}}$ and $\mathscr{A}_{\mu}$ is the purely bosonic part of the on-shell value of the auxiliary field $A_{\mu}$ given by

$$
\mathscr{A}_{\mu}=-i m_{\mathrm{P}}^{2}\left(D_{\mu} \Phi^{\alpha} \Omega_{\alpha}-D_{\mu} \Phi^{* \bar{\alpha}} \Omega_{\bar{\alpha}}\right) / 2 \Omega .
$$


It is clear from Eq. ([2.3]) that $S$ exhibits non-minimal couplings of the $\Phi^{\alpha}$, s to $R$. However, $\Omega$ enters the kinetic terms of the $\Phi^{\alpha}$ 's too. In general, $\Omega$ can be written as [7]

$$
-\Omega / 3=1-F_{K}\left(\Phi^{\alpha} \Phi^{* \bar{\alpha}}\right) / 3+\left(F_{R}\left(\Phi^{\alpha}\right)+F_{R}^{*}\left(\Phi^{* \bar{\alpha}}\right)\right)
$$

where $F_{K}$ is a dimensionless real function while $F_{R}$ is a dimensionless, holomorphic function. For $F_{R}>F_{K}, F_{K}$ expresses mainly the kinetic terms of the $\Phi^{\alpha}$ 's whereas $F_{R}$ represents the non-minimal coupling to gravity - note that $\Omega_{\alpha \bar{\beta}}$ is independent of $F_{R}$ since $F_{R, \Phi^{\alpha} \Phi^{* \bar{\beta}}}=0$. In order to get canonical kinetic terms, we need [8] $\mathscr{A}_{\mu}=0$ and $F_{K \alpha \bar{\beta}} \simeq \delta_{\alpha \bar{\beta}}$. The first condition is attained when the dynamics of the $\Phi^{\alpha}$ 's is dominated only by the real moduli $\left|\Phi^{\alpha}\right|$. The second condition is satisfied by the choice

$$
F_{K}\left(\left|\Phi^{\alpha}\right|^{2}\right)=\left|\Phi^{\alpha}\right|^{2} / m_{\mathrm{P}}^{2}+k_{\alpha \beta}\left|\Phi^{\alpha}\right|^{2}\left|\Phi^{\beta}\right|^{2} / m_{\mathrm{P}}^{4}
$$

with sufficiently small coefficients $k_{\alpha \beta}$. Here we assume that the $\Phi$ 's are charged under a global see Secs. [1] and [ - or gauge - see Sec. 目 - U(1) symmetry, so as mixed terms of the form $\Phi^{\alpha} \Phi_{\beta}^{*}$ are disallowed. The inclusion of the fourth order term for the accompanying non-inflaton field, $\Phi^{1}:=S$ is obligatory in order to evade [7] a tachyonic instability occurring along this direction. As a consequence, all the allowed terms are to be considered in the analysis for consistency.

\subsection{Modeling nMI in SUGRA}

The realization of nMI in SUGRA requires $\widehat{V}_{\mathrm{D}}=0$. This condition may be attained, when the inflaton is (the radial part of) a gauge:

- Singlet, by introducing an extra field $\Phi^{2}:=\Phi$ which obviously has zero contribution to $\widehat{V}_{\mathrm{D}}$.

- Non-singlet by introducing a conjugate pair of Higgs superfields, $\Phi^{2}:=\Phi$ and $\Phi^{3}:=\bar{\Phi}$, which are parameterized so as $\widehat{V}_{\mathrm{D}}=0$.

The presence of $S$ is crucial for the realization of our scenaria, since it assists us to isolate via its derivative the contribution of the inflaton(s) in $\widehat{V}$, Eq. (R.JC). Indeed, placing $S$ at the origin the resulting $\widehat{V}=\widehat{V}_{\mathrm{I} 0}-$ in both cases above - is equal to

$$
\widehat{V}_{\mathrm{I} 0}=e^{K / m_{\mathrm{P}}^{2}} K^{S S^{*}} W_{, S} W_{, S^{*}}^{*}=V_{\mathrm{F}} / f_{S \Phi} f_{R}^{2} \text { since } e^{K / m_{\mathrm{P}}^{2}}=1 / f_{R}^{3} \text { and } K^{S S^{*}}=f_{R} / f_{S \Phi},
$$

where $f_{R}=-\Omega / 3$ and $f_{S \Phi}=m_{\mathrm{P}}^{2} \Omega_{, S S^{*}}$. Also $V_{\mathrm{F}}=\left|W_{, S}\right|^{2}$ is the F-term SUSY potential, obtained from Eq. (R.IC) with $\mathrm{D}^{\alpha}=0$ in the limit $m_{\mathrm{P}} \rightarrow \infty$. Given that $f_{S \Phi} \ll f_{R}$, the construction of an inflationary plateau is reduced in the selection of the appropriate $W$ and $F_{R}$ so that $\widehat{V}_{\mathrm{I} 0} \simeq V_{\mathrm{F}} / f_{R}^{2}$ is almost constant. In the case of a gauge-singlet inflaton, where $\mathrm{D}^{\alpha}=0$, this objective can be achieved with one of the two choices:

- If we set $[2,8] W=\lambda S \Phi^{2}$ and $F_{R}=1+c_{R} \Phi^{2} / m_{\mathrm{P}}^{2}$ - cf. Sec. [1.3. - , we obtain $V_{\mathrm{F}}=\lambda^{2}|\Phi|^{4}$ and $f_{R}^{2} \simeq c_{R}^{2}|\Phi|^{4}$ for $c_{R} \gg 1$. Therefore, Eq. (L.7) implies that $\widehat{V}_{\mathrm{I} 0}$ turns out to be almost constant.

- If we choose [4] $W=m S \Phi$ and $F_{R}=1+c_{R} \Phi / m_{\mathrm{P}}$ - cf. Sec. [.3.2] -, we find $V_{\mathrm{F}}=m^{2}|\Phi|^{2}$ and $f_{R}^{2} \simeq c_{R}^{2}|\Phi|^{2}$ for $c_{R} \gg 1$. Eq. (2.]T) gives again an almost constant $\widehat{V}_{\mathrm{I} 0 \text {. }}$ 
In the case of a gauge non-singlet inflaton, we can take [3] $W=\lambda S\left(\Phi \bar{\Phi}-M^{2}\right)$ which can be combined with the gauge invariant $F_{R}, F_{R}=1+c_{R} \bar{\Phi} \Phi / m_{\mathrm{P}}^{2}-$ cf. Ref. [9]. For $|\Phi|=|\bar{\Phi}|$, we expect $V_{\mathrm{D}}=0, V_{\mathrm{F}} \simeq \lambda^{2}|\Phi|^{4}$ (for $|\Phi| \gg M$ ) and $f_{R}^{2} \simeq c_{R}^{2}|\Phi|^{4}$ for $c_{R} \gg 1$. Therefore, Eq. ([.]) again implies that $\widehat{V}_{\mathrm{I} 0}$ is almost constant. The GUT gauge group is spontaneously broken during and at the end of nMI, where $\Phi$ and $\bar{\Phi}$ acquire their vacuum expectation values (VEVs) $\langle\Phi\rangle=\langle\bar{\Phi}\rangle=M$ with SUSY unbroken - up to tiny corrections from SUSY breaking effects.

In the following we show details on the realization of these three scenaria. Our analysis is carried out exclusively in the EF substituting into Eq. ([2.Jd), Eqs. ([2.2) and ([2.5) and the chosen $F_{R}$. Then, Eq. (년) can be employed in order to find the canonically normalized inflaton field $\widehat{\phi}$ in terms of $\phi$ defining

$$
f_{K} \dot{\phi}^{2} / 2=m_{\mathrm{P}}^{2} \Omega_{\alpha \bar{\beta}} \dot{\Phi}^{\alpha} \dot{\Phi}^{* \bar{\beta}} \text { and } f_{R}=-\Omega / 3 \text {. }
$$

We also check the stability of the inflationary trajectory w.r.t the fluctuations of the non-inflaton fields and find the whole spectrum during nMI. Employing the derived masses we find the one-loop radiative correction $\Delta V$ and the corresponding EF potential using the Coleman-Weinberg formula

$$
\widehat{V}_{\mathrm{I}}=\widehat{V}_{\mathrm{I} 0}+\Delta V \text { with } \Delta V=\frac{1}{64 \pi^{2}} \sum_{i}(-)^{\mathrm{F}_{i}} M_{i}^{4} \ln \frac{M_{i}^{2}}{\Lambda^{2}},
$$

where the sum extends over all helicity states $i, \mathrm{~F}_{i}$ and $M_{i}^{2}$ is the fermion number and mass squared of the $i$ th state and $\Lambda$ is a renormalization mass scale.

\section{3. nMI with Quartic Potential for a Gauge Singlet Inflaton}

This setting is realized in the presence of two superfields $S$ and $\Phi$ charged under a global $U(1)$ symmetry with charges 2 and -1 respectively. In particular we take

$$
W=\lambda S \Phi^{2}, F_{R}=\frac{c_{\Phi}}{4 m_{\mathrm{P}}^{2}} \Phi^{2}, F_{K}=\frac{|S|^{2}}{m_{\mathrm{P}}^{2}}+\frac{|\Phi|^{2}}{m_{\mathrm{P}}^{2}}-2 k_{S} \frac{|S|^{4}}{m_{\mathrm{P}}^{4}}-2 k_{\Phi} \frac{|\Phi|^{4}}{m_{\mathrm{P}}^{4}}-2 k_{S \Phi} \frac{|S|^{2}|\Phi|^{2}}{m_{\mathrm{P}}^{4}} .
$$

In Sec. B.] we describe the salient features of this model and in Sec. B.2] we expose our results.

\subsection{Structure of the Inflationary Potential}

The EF F-term (tree level) SUGRA scalar potential, $\widehat{V}$, of this model is obtained from Eq. (R.TC)

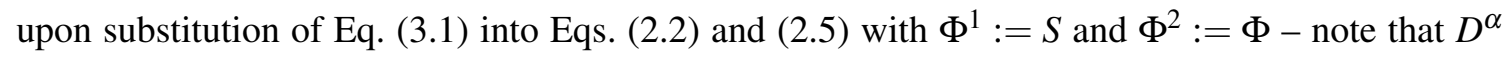
vanish by construction. We can verify that along the trajectory

$$
S=0 \text { and } \theta:=\arg \Phi=0
$$

and for $c_{\Phi} \gg 1, \widehat{V}$ develops a plateau with almost constant potential energy density, $\widehat{V}_{\mathrm{I} 0}$ and corresponding Hubble parameter $\widehat{H}_{\mathrm{I}}$ found by Eq. (2.7):

$$
\widehat{V}_{\mathrm{I} 0}=\frac{\lambda^{2} \phi^{4}}{4 f_{S \Phi} f_{R}^{2}} \simeq \frac{\lambda^{2} m_{\mathrm{P}}^{4}}{4 f_{S \Phi} c_{R}^{2}} \text { and } \widehat{H}_{\mathrm{I}}=\frac{\widehat{V}_{\mathrm{I} 0}^{1 / 2}}{\sqrt{3} m_{\mathrm{P}}} \simeq \frac{\lambda m_{\mathrm{P}}}{2 \sqrt{3} f_{S \Phi} c_{R}}
$$

where $f_{R}$ and $f_{S \Phi}$ are calculated by employing their definitions below Eq. ([2.])

$$
f_{R}=1+c_{R} x_{\phi}^{2}+k_{\Phi} x_{\phi}^{4} / 6 \text { and } f_{S \Phi}=1-k_{S \Phi} x_{\phi}^{2} \text { with } x_{\phi}=\phi / m_{\mathrm{P}} \text { and } c_{R}=c_{\Phi} / 4-1 / 6
$$




\begin{tabular}{|c|c|l|}
\hline FIELDS & EINGESTATES & \multicolumn{1}{|c|}{ MASS SQUARED } \\
\hline \hline 1 real scalar & $\widehat{\theta}$ & $m_{\widehat{\theta}}^{2}=\lambda^{2}\left(1+6 c_{R}\right) m_{\mathrm{P}}^{2} x_{\phi}^{4} / 3 f_{S \Phi} f_{R}^{3} J^{2} \simeq 4 \widehat{H}_{\mathrm{I}}^{2}$ \\
2 real scalars & $\widehat{s}_{1}, \widehat{s}_{2}$ & $m_{\widehat{s}}^{2} \simeq \lambda^{2} m_{\mathrm{P}}^{2}\left(2+c_{R}^{2}\left(12 k_{S}\left(1+c_{R} x_{\phi}^{2}\right)-1\right) x_{\phi}^{4}\right) / 6 c_{R}^{2} f_{S \Phi}^{3} f_{R}^{2}$ \\
\hline 2 Weyl spinors & $\widehat{\psi}_{ \pm}=\frac{\widehat{\psi}_{\Phi} \pm \widehat{\psi}_{S}}{\sqrt{2}}$ & $m_{\widehat{\psi} \pm}^{2} \simeq \lambda^{2} m_{\mathrm{P}}^{2}\left(2-k_{S \Phi} x_{\phi}^{2}+k_{S \Phi} c_{R} x_{\phi}^{4}\right)^{2} / 18 f_{S \Phi}^{3} f_{R}^{2}$ \\
\hline
\end{tabular}

Table 1: The mass spectrum of the model along the inflationary trajectory of Eq. (3.2).

Expanding $\Phi$ and $S$ in real and imaginary parts according to the prescription

$$
\Phi=\phi e^{i \theta} / \sqrt{2} \text { and } S=\left(s_{1}+i s_{2}\right) / \sqrt{2},
$$

we find that along the trajectory of Eq. (B.2), $K_{\alpha \bar{\beta}}$ defined in Eq. (2.16) takes the form

$$
\left(K_{\alpha \bar{\beta}}\right)=\operatorname{diag}\left(J^{2}, f_{S \Phi} / f_{R}\right) \text { where } J \simeq \sqrt{3} c_{R}^{2} \phi^{2} / \sqrt{2} f_{R}^{2} \simeq \sqrt{6} / x_{\phi},
$$

as can be inferred from Eq. (ㄷ.4) for $f_{K}=1-4 k_{\Phi} x_{\phi}^{2}-$ see Eq. (ㄷ.8). Consequently, we can introduce the EF canonically normalized fields, $\widehat{\phi}, \widehat{\theta}$ and $\widehat{s_{i}}$, with $i=1,2$ as follows - cf. Ref. [2,3,7,8]:

$$
K_{\alpha \bar{\beta}} \dot{\Phi}^{\alpha} \dot{\Phi}^{* \bar{\beta}}=\frac{1}{2}\left(\dot{\hat{\phi}}^{2}+\dot{\hat{\theta}}^{2}+\dot{\hat{s}}_{1}^{2}+\dot{\hat{s}}_{2}^{2}\right)
$$

where the dot denotes derivation w.r.t the JF cosmic time and the hatted fields are defined as follows

$$
d \widehat{\phi} / d \phi=J, \widehat{\theta} \simeq J \phi \theta \text { and } \widehat{s_{i}}=\sqrt{f_{S \Phi} / f_{R}} s_{i} \text { with } i=1,2 .
$$

To check the stability of $\widehat{V}$ in Eq. (R.JC) along the trajectory in Eq. (B.2) w.r.t the fluctuations

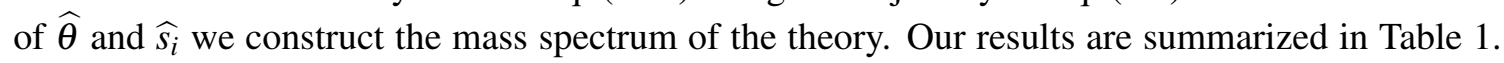
From there it is evident that $k_{S} \gtrsim 0.5$ assists us to achieve $m_{\widehat{s}}^{2}>0$. We have also numerically verified that the various masses remain greater than $\widehat{H}_{\mathrm{I}}$ during the last 50 e-foldings of $\mathrm{nMI}$, and so any inflationary perturbations of the fields other than the inflaton are safely eliminated. In Table $\square$ we also present the masses squared of chiral fermions along the direction of Eq. (4.3]), which can be served for the calculation of $\Delta V$ in Eq. (2. $)$. We observe that the fermionic (4) and bosonic (4) degrees of freedom (d.o.f) are equal - here we take into account the 1 d.o.f of $\widehat{\phi}$ which is not perturbed. $\Delta V$ has no impact on our results, since the slope of the inflationary path is generated at the classical level and the various masses are proportional to the weak coupling $\lambda$.

\subsection{Results}

As can be easily seen from the relevant expressions above, the model depends on the following parameters: $\lambda, k_{S}, k_{\Phi}, k_{S \Phi}$, and $c_{R}$. Recall that we use $T_{\mathrm{rh}}=10^{8} \mathrm{GeV}$ throughout. Our results are essentially independent of $k_{S}$, provided that $m_{\widehat{S}}^{2}>0$ for $\lambda<1-$ see in Table $⿴$. We therefore set $k_{S}=0.5$. Also we take a central value $k_{\Phi}=0.5$. Besides these two values, in our numerical code, we use as input parameters $c_{R}, k_{S \Phi}$ and $\phi_{*}$. For every chosen $c_{R} \geq 1$, we restrict $\lambda$ and $\phi_{*}$ so that the 


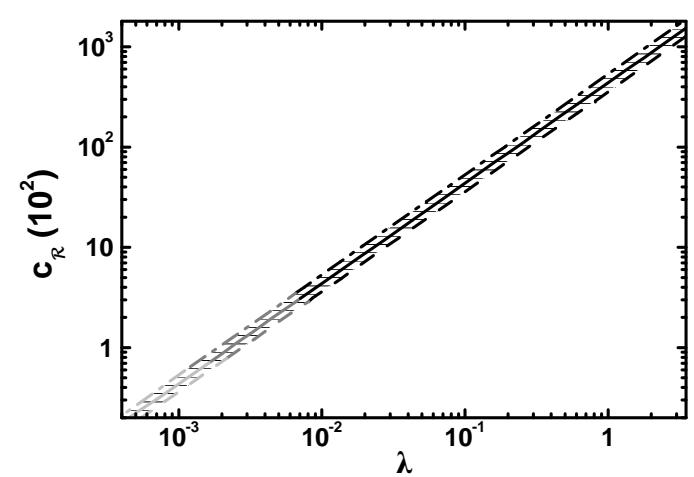

(a)

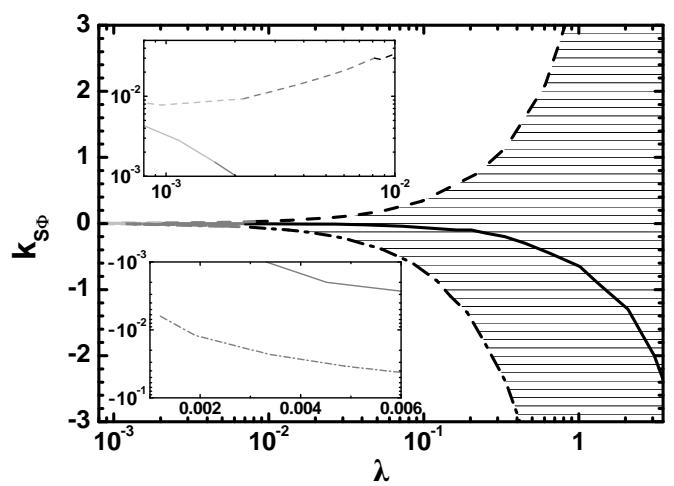

(b)

Figure 2: Allowed (hatched) region as determined by Eqs. (1.5), (1.7) and (1.9) in the $\lambda-c_{R}\left[\lambda-k_{S \Phi}\right]$ plane (a) [(b)], for $k_{S}=k_{\Phi}=0.5$. The conventions adopted for the type and the color of the various lines are described in Fig. 1.

conditions Eqs. (ㄷ.5) and (ㄷ.7) are satisfied. By adjusting $k_{S \Phi}$ we can achieve $n_{\mathrm{s}}$ 's in the range of Eq. (ㅁ. ). Our results are displayed in Fig. ㅁ-(a) [Fig. (-(b)], where we delineate the hatched region

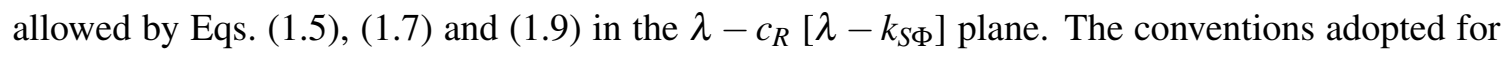
the various lines are shown in Fig. $\square$. In particular, the dashed [dot-dashed] lines correspond to $n_{\mathrm{s}}=0.975\left[n_{\mathrm{s}}=0.946\right]$, whereas the solid lines are obtained by fixing $n_{\mathrm{s}}=0.96-$ see Eq. (L.T).

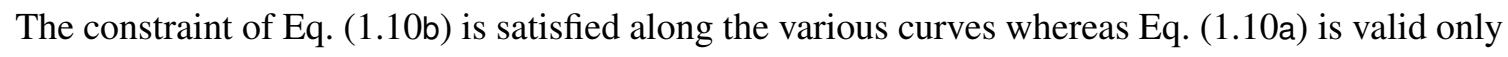
along the gray and light gray segments of these. Along the light gray segments, though, we obtain $\phi_{*} \geq m_{\mathrm{P}}$. Note that for vanishing $k_{S \Phi}$ and $k_{\Phi}$ our results can be approximated by the analytical expressions exhibited in the paragraph (ii) of Sec. [.3.] replacing $\sqrt{\lambda}$ with $\lambda$. Indeed, $c_{R}$ remains almost proportional to $\lambda$ and for constant $\lambda, c_{R}$ increases as $n_{\mathrm{s}}$ decreases. We remark that mostly negative $k_{S \Phi}$ 's are needed which for $\lambda>0.16 \Leftrightarrow \phi_{*}<0.1 m_{\mathrm{P}}-$ see Eq. ([.]2d) - take quite natural (of order one) values. Focusing on $k_{S \Phi}<0$ for $n_{\mathrm{s}}=0.96$ and $\widehat{N}_{*} \simeq 50$ we find

$$
112 \lesssim c_{R} \lesssim 1.6 \cdot 10^{5} \text { with } 2.5 \cdot 10^{-3} \lesssim \lambda \lesssim 3.7 \text { and } 0 \lesssim-k_{S \Phi} \lesssim 2.5
$$

Also $6.8 \lesssim\left|\alpha_{\mathrm{s}}\right| / 10^{-4} \lesssim 8.2$ and $r \simeq 3.8 \cdot 10^{-3}$ which lie within the allowed ranges of Eq. (매).

\section{4. nMI with Quartic Potential for a Gauge non-Singlet Inflaton}

In the present scheme the inflaton field can be identified with the radial component of a conjugate pair of Higgs superfields. We here - cf. Ref. [9] - focus on the Higgs superfields, $\bar{\Phi}$ and $\Phi$, with $B-L=-1,1$ which break a GUT symmetry, e.g., $G_{\mathrm{GUT}}=G_{\mathrm{SM}} \times U(1)_{B-L}$ down to MSSM gauge group $G_{S M}$ through their VEVs. We also impose a $U(1)$ R symmetry, $U(1)_{R}$, which guarantees the linearity of the superpotential, $W$, w.r.t the singlet $S$ - see Table $\square$. The functions $W, F_{R}$

\begin{tabular}{|l||ccc|}
\hline SUPERFIELDS & $S$ & $\Phi$ & $\bar{\Phi}$ \\
\hline \hline$U(1)_{B-L}$ & 0 & 1 & -1 \\
\hline$R$ & 1 & 0 & 0 \\
\hline
\end{tabular}

Table 2: Charge assignments of the superfields. 
and $F_{K}$, which respect the imposed symmetries, are respectively given by

$$
\begin{aligned}
W & =\lambda S\left(\bar{\Phi} \Phi-M^{2}\right), F_{R}=\frac{c_{\bar{\Phi} \Phi}}{2 m_{\mathrm{P}}^{2}} \bar{\Phi} \Phi \\
F_{K} & =\frac{|S|^{2}}{m_{\mathrm{P}}^{2}}+\frac{|\Phi|^{2}}{m_{\mathrm{P}}^{2}}+\frac{|\bar{\Phi}|^{2}}{m_{\mathrm{P}}^{2}}-k_{S} \frac{|S|^{4}}{m_{\mathrm{P}}^{4}}-k_{\Phi} \frac{|\Phi|^{4}}{m_{\mathrm{P}}^{4}}-\bar{k}_{\Phi} \frac{|\bar{\Phi}|^{4}}{m_{\mathrm{P}}^{4}} \\
& -2 k_{S \Phi} \frac{|S|^{2}|\Phi|^{2}}{m_{\mathrm{P}}^{4}}-2 \bar{k}_{S \Phi} \frac{|S|^{2}|\bar{\Phi}|^{2}}{m_{\mathrm{P}}^{4}}-2 k_{\Phi \bar{\Phi}} \frac{|\Phi|^{2}|\bar{\Phi}|^{2}}{m_{\mathrm{P}}^{4}}
\end{aligned}
$$

We below outline the salient features of our inflationary scenario (Sec. A. I) and then, we present its predictions in Sec. 4.2.

\subsection{Structure of the Inflationary Potential}

The EF F-term (tree level) SUGRA scalar potential, $\widehat{V}_{\mathrm{I} 0}$, for this model is obtained by substi-

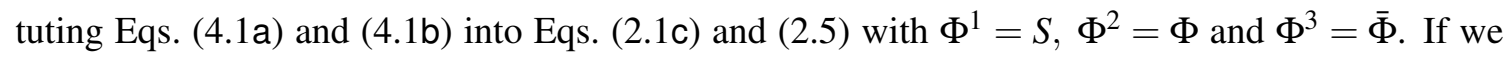
parameterize the SM neutral fields $\Phi$ and $\bar{\Phi}$ by

$$
\Phi=\phi e^{i \theta} \cos \theta_{\Phi} / \sqrt{2} \text { and } \bar{\Phi}=\phi e^{i \bar{\theta}} \sin \theta_{\Phi} / \sqrt{2},
$$

we can easily deduce that a D-flat direction occurs at

$$
\theta=\bar{\theta}=0 \text { and } \theta_{\Phi}=\pi / 4
$$

provided that $K$ remains invariant under the interchange $\Phi \rightarrow \bar{\Phi}$ and $\bar{\Phi} \rightarrow \Phi$, which implies

$$
k_{\Phi}=\bar{k}_{\Phi} \text { and } k_{S \Phi}=\bar{k}_{S \Phi}
$$

From Eq. (R.TC), we can verify that for $c_{R} \gg 1, k_{S \Phi} \ll 1$ and $m_{B L} \ll 1, \widehat{V}$ takes a form suitable for the realization of nMI, since it develops a plateau. The (almost) constant potential energy density $\widehat{V}_{\mathrm{I} 0}$ and the corresponding Hubble parameter $\widehat{H}_{\mathrm{I}}$ - along the trajectory in Eq. (4.3) - are given by

$$
\widehat{V}_{\mathrm{I} 0}=m_{\mathrm{P}}^{4} \frac{\lambda^{2}\left(x_{\phi}^{2}-4 m_{B L}^{2}\right)^{2}}{16 f_{S \Phi} f_{R}^{2}} \simeq \frac{\lambda^{2} m_{\mathrm{P}}^{4}}{16 f_{S \Phi} c_{R}^{2}} \text { and } \widehat{H}_{\mathrm{I}}=\frac{\widehat{V}_{\mathrm{I} 0}^{1 / 2}}{\sqrt{3 f_{S \Phi}} m_{\mathrm{P}}} \simeq \frac{\lambda m_{\mathrm{P}}}{4 \sqrt{3 f_{S \Phi}} c_{R}},
$$

where $f_{R}$ and $f_{S \Phi}$, given by their definitions below Eq. (‥Z), are specified as follows

$$
f_{R}=1+c_{R} x_{\phi}^{2}+\left(k_{\Phi}+k_{\Phi \bar{\Phi}}\right) \frac{x_{\phi}^{4}}{24}, f_{S \Phi}=1-k_{S \Phi} x_{\phi}^{2} \quad \text { with } \quad m_{B L}=\frac{M}{m_{\mathrm{P}}} \text { and } c_{R}=\frac{c_{\bar{\Phi} \Phi}}{4}-\frac{1}{6} .
$$

We next proceed to check the stability of the trajectory in Eq. (4.3]) w.r.t the fluctuations of $\theta_{\Phi}, \theta, \bar{\theta}$ and $S=s / \sqrt{2}$ - here $S$ has been rotated to the real axis via a suitable R transformation. We first check that $\widehat{V}_{, \chi}=0$, with $\chi=\theta_{\Phi}, \theta, \bar{\theta}$ and $s$, and then we find

$$
\begin{aligned}
K_{\alpha \bar{\beta}} \dot{\Phi}^{\alpha} \dot{\Phi}^{* \bar{\beta}} & =\frac{1}{2} J^{2}\left(\dot{\phi}^{2}+\frac{1}{2} \phi^{2} \dot{\theta}_{+}^{2}\right)+\frac{f_{\Phi} \phi^{2}}{2 f_{R}}\left(\frac{1}{2} \dot{\theta}_{-}^{2}+\dot{\theta}_{\Phi}^{2}\right)+\frac{f_{S \Phi}}{2 f_{R}} \dot{s} \\
& =\frac{1}{2}\left(\dot{\hat{\phi}}^{2}+\dot{\hat{\theta}}_{+}^{2}+\dot{\hat{\theta}}_{-}^{2}+\dot{\hat{\theta}}_{\Phi}^{2}+\dot{\vec{s}}^{2}\right) \text { with } \theta_{ \pm}=\frac{\bar{\theta} \pm \theta}{\sqrt{2}} \text { and } s=\frac{S}{\sqrt{2}}
\end{aligned}
$$




\begin{tabular}{|c||c|l|}
\hline FIELDS & EIGENSTATES & \multicolumn{1}{|c|}{ MASSES SQUARED } \\
\hline \hline 2 real scalars & $\widehat{\theta}_{\Phi}$ & $m_{\widehat{\theta}_{\Phi}}^{2} \simeq g^{2} m_{\mathrm{P}}^{2} x_{\phi}^{2} / 4 f_{\Phi} f_{R}$ \\
& $\widehat{\boldsymbol{\theta}}_{+}$ & $m_{\widehat{\theta}_{+}}^{2}=\lambda^{2} m_{\mathrm{P}}^{2} x_{\phi}^{6} c_{R} / 12 f_{R}^{3} f_{S \Phi} \simeq 4 \widehat{H}_{\mathrm{I}}^{2}$ \\
& $\widehat{S}$ & $m_{\widehat{S}}^{2}=\lambda^{2} m_{\mathrm{P}}^{2}\left(12+x_{\phi}^{2}\left(1+6 c_{R}^{2} x_{\phi}^{2}\right)\left(6 k_{S}-1\right)\right.$ \\
& & $\left.+36 c_{R}^{3} k_{S} x_{\phi}^{6}\right) / 144 c_{R}^{2} f_{S \Phi}^{3} f_{R}^{2}$ \\
\hline 1 gauge boson & $A_{B L}$ & $M_{B L}^{2}=g^{2} m_{\mathrm{P}}^{2} x_{\phi}^{2} f_{\Phi} / 4 f_{R}$ \\
\hline 4 Weyl spinors & $\widehat{\psi}_{ \pm}=\frac{\widehat{\psi}_{\Phi+} \pm \widehat{\psi}_{S}}{\sqrt{2}}$ & $m_{\widehat{\psi}_{ \pm}}^{2} \simeq \lambda^{2} m_{\mathrm{P}}^{2}\left(2+k_{S \Phi} x_{\phi}^{2}\left(c_{R} x_{\phi}^{2}-1\right)\right) / 36 f_{S \Phi}^{3} f_{R}^{2} c_{R}^{2}$ \\
& $\lambda_{B L}, \widehat{\psi}_{\Phi-}$ & $M_{B L}^{2}=g^{2} m_{\mathrm{P}}^{2} x_{\phi}^{2} f_{\Phi} / 4 f_{R}$ \\
\hline
\end{tabular}

Table 3: The mass spectrum of the model along the inflationary trajectory of Eq. (4.3) in the presence of the conditions in Eq. (4.4). To avoid very lengthy formulas we neglect terms proportional to $m_{B L}^{2} \ll x_{\phi}^{2}$.

In the last line, we introduce the EF canonically normalized fields, $\widehat{\phi}, \widehat{\theta}_{+}, \widehat{\theta}_{-}, \widehat{\theta}_{\Phi}$ and $\widehat{s}$, defined as follows - cf. Ref. [3]:

$$
\frac{d \widehat{\phi}}{d \phi}=J, \widehat{\theta}_{+}=\frac{J \phi \theta_{+}}{\sqrt{2}}, \widehat{\theta}_{-}=\sqrt{\frac{f_{\Phi}}{2 f_{R}}} \phi \theta_{-}, \widehat{\theta}_{\Phi}=\sqrt{\frac{f_{\Phi}}{f_{R}}} \phi\left(\theta_{\Phi}-\frac{\pi}{4}\right) \text { and } \widehat{s}=\sqrt{\frac{f_{S \Phi}}{f_{R}}} s,
$$

where $J$ and $f_{K}$ can be found from Eqs. ([L]) and (2.8) with $f_{\Phi}=1-\left(k_{\Phi}+k_{\Phi \bar{\Phi}}\right) x_{\phi}^{2}$.

Having defined the canonically normalized scalar fields, we can derive the mass spectrum of the model along the direction of Eq. (4.3)). Our results are listed in Table B B, where we present the eigenvalues and the corresponding eigenvectors of the relevant mass-squared matrices. As we observe, no instability arises in the spectrum, since $k_{S} \gtrsim 1$ ensures $m_{\widehat{S}}^{2}>0$ and $m_{\widehat{\theta}_{\Phi}}^{2}>0$ thanks the D-term contributions which are proportional to $g \simeq 0.7>\lambda$. Moreover, the masses of the various scalars remain greater than $\widehat{H}_{\mathrm{I}}$ and so any perturbations of the fields other than the inflaton are safely eliminated. We also remark that $U(1)_{B-L}$ is broken during nMI and therefore the gauge boson $A_{B L}$ becomes massive absorbing the massless Goldstone boson associated with $\widehat{\theta}_{-}$. As a consequence, no cosmic strings are produced at the end of nMI and so, no extra restrictions on the parameters have to be imposed. From Table B] we can deduce that the numbers of bosonic (8) and fermionic (8) d.o.fs are equal. Plugging these results into Eq. (2.9) we get $\Delta V$, which is dominated by the contributions from $m_{\widehat{\theta}_{\Phi}}$ and $M_{B L}$ since these are proportional to $g \gg \lambda$. Namely we find

$$
\Delta V \simeq \frac{1}{64 \pi^{2}}\left(m_{\widehat{\theta}_{\Phi}}^{4} \ln \left(m_{\widehat{\theta}_{\Phi}}^{2} / \Lambda^{2}\right)-M_{B L}^{4} \ln \left(M_{B L}^{2} / \Lambda^{2}\right)\right)
$$

Note that the presence of $f_{\Phi} \neq 1$ prevents the exact cancellation, occurring in Ref. [3], of the two contributions above. Since this result can be continued until the SUSY vacuum of the theory, we determine there the employed $\Lambda$ by imposing the condition $\Delta V=0$.

\subsection{Results}

The free parameters of this model are $\lambda, M, k_{S}, k_{\Phi}, k_{\Phi \bar{\Phi}}, k_{S \Phi}$, and $c_{R}$. Following the same reasoning with Sec. B.2] we set $k_{S}=1$ and $k_{\Phi}=0.1$ throughout. Moreover, we can determine $M$ 


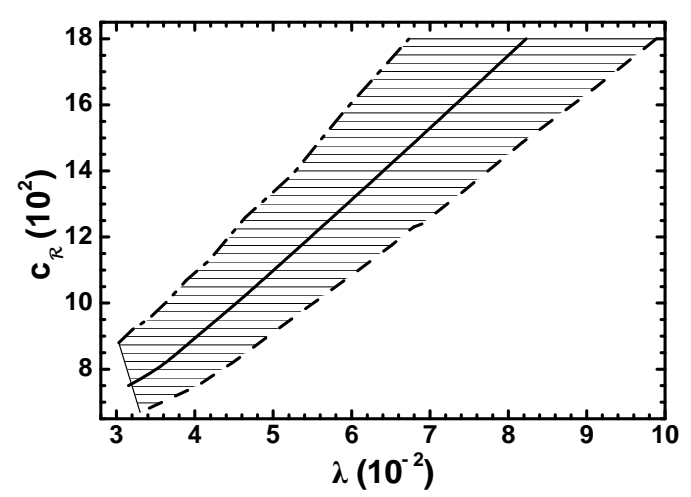

(a)

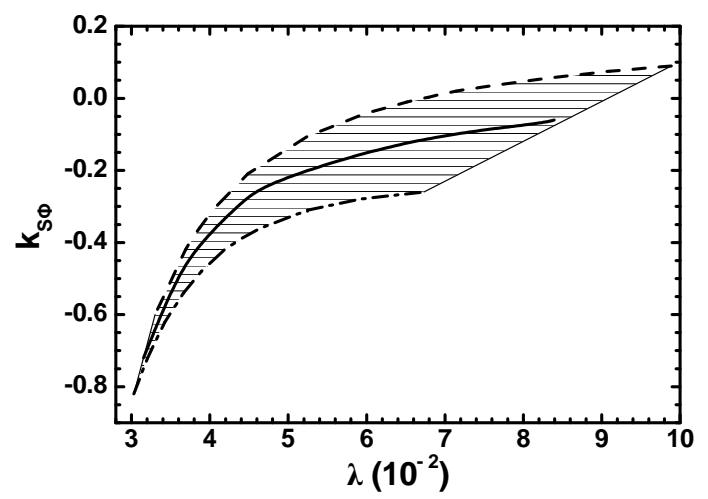

(b)

Figure 3: Allowed (hatched) region as determined by Eqs. (1.5), (1.7) and (1.9) in the $\lambda-c_{R}\left[\lambda-k_{S \Phi}\right]$ plane (a) [(b)] for $k_{S}=1, k_{\Phi \bar{\Phi}}=0.1$ and $k_{\Phi}=0.01$. The conventions adopted are described in Fig. 1 .

identifying the mass of the gauge boson $A_{B L}$ in the SUSY vacuum with the GUT scale $M_{\mathrm{GUT}} \simeq$ $2 \cdot 10^{16} \mathrm{GeV}$ within the MSSM, i.e.

$$
\sqrt{f_{\Phi 0} / f_{R 0}} g M=M_{\mathrm{GUT}} \Rightarrow m_{B L} \simeq\left(2 \sqrt{2 c_{R}^{\max }-c_{R}}\right)^{-1} \text { with } c_{R}^{\max }=g^{2} m_{\mathrm{P}}^{2} / 8 M_{\mathrm{GUT}}^{2},
$$

$f_{R 0}=f_{R}\left(x_{\phi}=2 m_{B L}\right)$ and $f_{\Phi 0}=f_{\Phi}\left(x_{\phi}=2 m_{B L}\right)$. The requirement $2 c_{R}^{\max }-c_{R}>0$ sets an upper bound $c_{R}<2 c_{R}^{\max } \simeq 1.8 \cdot 10^{3}$. To obtain solutions consistent with the requirements of Sec. $\square 2$ we need a rather low $k_{\Phi}$. We take $k_{\Phi}=0.01$.

Working along the lines of Sec. B.2] we delineate the allowed (hatched) region, as determined

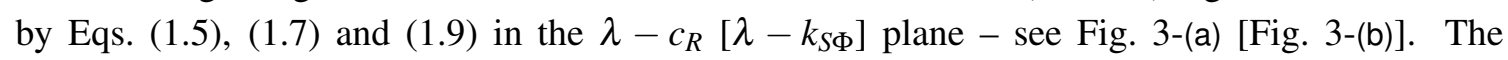
conventions adopted for the various lines are shown in Fig. $\mathrm{W}$. We observe that the allowed region is considerably shrunk w.r.t that obtained with a gauge singlet inflaton in Fig. \ and this is limited in the regime where only Eq. ( $(\mathbb{L} \cdot \mathbf{l} \mathrm{b})$ is valid. Also $c_{R}$ remains almost proportional to $\lambda$ and increases as $n_{\mathrm{s}}$ decreases. Note that for vanishing $k$ 's, our results can be approximated by the analytical expressions exhibited in the paragraph (ii) of Sec. …] replacing $\sqrt{\lambda}$ with $\lambda / 2$. Especially for $n_{\mathrm{s}}=0.96$ and $\widehat{N}_{*} \simeq 50$ we find

$$
7.3 \lesssim c_{R} / 10^{2} \lesssim 18 \text { with } 2.9 \lesssim \lambda / 10^{-2} \lesssim 8.4 \text { and } 0.6 \lesssim-k_{S \Phi} / 10^{-1} \lesssim 8.5 \text {. }
$$

Therefore, $k_{S \Phi}<0$ assists us again to obtain the central $n_{\mathrm{S}}$ in Eq. ([L.9a). Also $0.7 \lesssim\left|\alpha_{\mathrm{s}}\right| / 10^{-3} \lesssim 1.2$ and $r \simeq(3-3.8) \cdot 10^{-3}$ in agreement with Eq. (ㅍ) .

\section{5. nMI with Quadratic Potential}

This model [4] is characterized by the following ingredients:

$$
W=m S \Phi, F_{R}=\frac{c_{\Phi}}{\sqrt{2} m_{\mathrm{P}}} \Phi, F_{K}=\frac{|S|^{2}}{m_{\mathrm{P}}^{2}}+\frac{|\Phi|^{2}}{m_{\mathrm{P}}^{2}}-2 k_{S} \frac{|S|^{4}}{m_{\mathrm{P}}^{4}}-2 k_{\Phi} \frac{|\Phi|^{4}}{m_{\mathrm{P}}^{4}}-2 k_{S \Phi} \frac{|S|^{2}|\Phi|^{2}}{m_{\mathrm{P}}^{4}}
$$

To ensure the form of $W$ and $F_{K}$ we can impose a global $U(1)$ symmetry under which $S$ and $\Phi$ have charges 1 and -1 . The imposed $U(1)$ is broken though during nMI by $F_{R}$. Due to the form of $F_{R}$, in which $\Phi$ appears linearly, $\Phi$ has to be singlet under the gauge group of the theory and therefore, we obtain $\mathrm{D}^{\alpha}=0$ by construction. In Sec. 5.] we outline the derivation of the inflationary potential of this model and in Sec. 5.2 we present our results. 


\begin{tabular}{|c|c|c|}
\hline FIELDS & EINGESTATES & MASSES SQUARED \\
\hline \hline 1 real scalar & $\widehat{\theta}$ & $m_{\widehat{\theta}}^{2} \simeq c_{R} m^{2} x_{\phi} / f_{R}^{3} J^{2} \simeq 4 \widehat{H}_{\mathrm{I}}^{2}$ \\
2 real scalars & $\widehat{s}_{1}, \widehat{s}_{2}$ & $m_{\widehat{s}}^{2} \simeq m^{2}\left(2+c_{R}^{2} x_{\phi}^{2}\left(12 c_{R} k_{S}-1\right)\right) / f_{S \Phi}^{3} f_{R}^{2}\left(2+3 c_{R}^{2}\right)$ \\
\hline 2 Weyl spinors & $\widehat{\psi}_{ \pm}=\frac{\widehat{\psi}_{\Phi} \pm \widehat{\psi}_{S}}{\sqrt{2}}$ & $m_{\widehat{\psi} \pm}^{2} \simeq m^{2}\left(6+x_{\phi}^{2}+6 c_{R} k_{S \Phi} x_{\phi}^{3}\right)^{2} / 12 f_{S \Phi}^{3} f_{R}^{2}\left(2+3 c_{R}^{2}\right)$ \\
\hline
\end{tabular}

Table 4: The mass spectrum of the model along the inflationary trajectory in Eq. (3.2).

\subsection{Structure of the Inflationary Potential}

Inserting Eq. ([ل Eq. (B.2) for $c_{R} \gg 1$ and $x_{\phi} \ll \sqrt{6}$, develops a plateau with (almost) constant potential energy density, $\widehat{V}_{\mathrm{I} 0}$ and corresponding Hubble parameter $\widehat{H}_{\mathrm{I}}$ calculated, in accordance with Eq. (2.]), as

$$
\widehat{V}_{\mathrm{I} 0}=\frac{m^{2} \phi^{2}}{2 f_{S \Phi} f_{R}^{2}} \simeq \frac{m^{2} m_{\mathrm{P}}^{2}}{2 c_{R}^{2} f_{S \Phi}} \text { and } \widehat{H}_{\mathrm{I}}=\frac{\widehat{V}_{\mathrm{I} 0}^{1 / 2}}{\sqrt{3} m_{\mathrm{P}}} \simeq \frac{m}{\sqrt{6 f_{S \Phi}} c_{R}},
$$

where $f_{R}$ and $f_{S \Phi}$, defined below Eq. (2.7), are specified as follows

$$
f_{R}=1+c_{R} x_{\phi}-x_{\phi}^{2} / 6+k_{\Phi} x_{\phi}^{4} / 6 \text { and } f_{S \Phi}=1-k_{S \Phi} x_{\phi}^{2} .
$$

To check the stability of the trajectory of Eq. (B.2) w.r.t the fluctuations of the various fields we expand $\Phi$ and $S$ in real and imaginary parts according to the prescription of Eq. (B.4). We then find that along the trajectory of Eq. (B.2), $K_{\alpha \bar{\beta}}$ defined in Eq. ([2.J6) turns out to be

$$
\left(K_{\alpha \bar{\beta}}\right)=\operatorname{diag}\left(J^{2}, f_{S \Phi} / f_{R}\right) \text { where } J \simeq \sqrt{3} c_{R} / \sqrt{2} f_{R} \simeq \sqrt{3 / 2} x_{\phi}^{-1} .
$$

Consequently, we can introduce the EF canonically normalized fields, $\widehat{\phi}, \widehat{\theta}$ and $\widehat{s_{i}}$, with $i=1,2$ which satisfy Eq. (B.6) with the hatted fields defined as in Eq. (B.7). As in the previous cases, we check the stability of the inflationary path in Eq. (B.2) deriving the mass spectrum of the model, presented in Table 6 . We observe that now the various masses are proportional to $m$ and obviously our findings are similar to those obtained in Sec. B.d.

\subsection{Results}

This model depends on the parameters: $m, k_{S}, k_{S \Phi}, k_{\Phi}$, and $c_{R}$. Following the same reasoning with Sec. B.2 we set throughout $k_{S}=k_{\Phi}=0.5$. The allowed (hatched) regions in the $m-c_{R}$ and

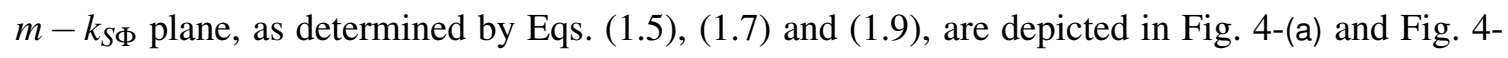
(b) respectively, following the conventions Fig. W. We confine conservatively ourselves to values $m \lesssim 3 \cdot 10^{17} \mathrm{GeV}$. For vanishing $k$ 's, our results here are almost identical with those in paragraph (ii) of Sec. प3.2. For $m \gtrsim 4 \cdot 10^{16} \mathrm{GeV}$, rather natural $k_{S \Phi}$ 's result to $n_{\mathrm{s}}=0.96$. For this $n_{\mathrm{s}}, \widehat{N}_{*} \simeq 50$ and confining ourselves to $k_{S \Phi}<0$ we find

$$
1.2 \lesssim c_{R} / 10^{2} \lesssim 60 \text { with } 0.5 \lesssim m / 10^{16} \mathrm{GeV} \lesssim 3 \text { and } 0 \lesssim-k_{S \Phi} \lesssim 2.4
$$

Also $\alpha_{\mathrm{s}} \simeq-6.5 \cdot 10^{-4}$ and $r \simeq 3.9 \cdot 10^{-3}$ compatibly with Eq. (ㅍ. $)$. Note, though, that for $k_{S \Phi}>0$ and $\phi>m_{\mathrm{P}}$ we can obtain $\alpha_{\mathrm{s}}$ and $r$ as large as 0.006 and 0.036 respectively. 


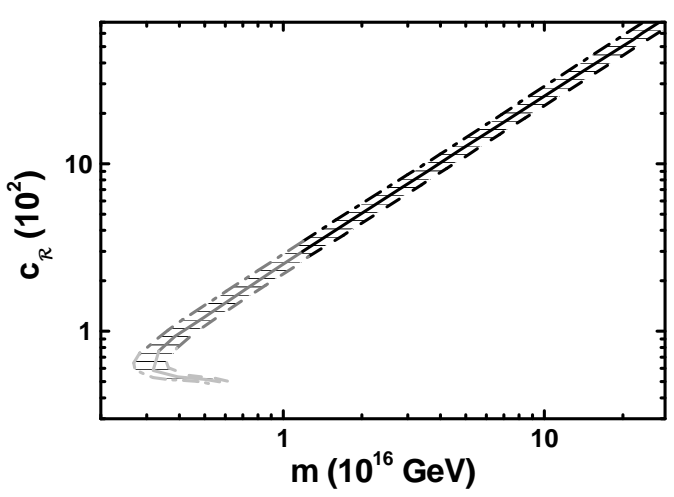

(a)

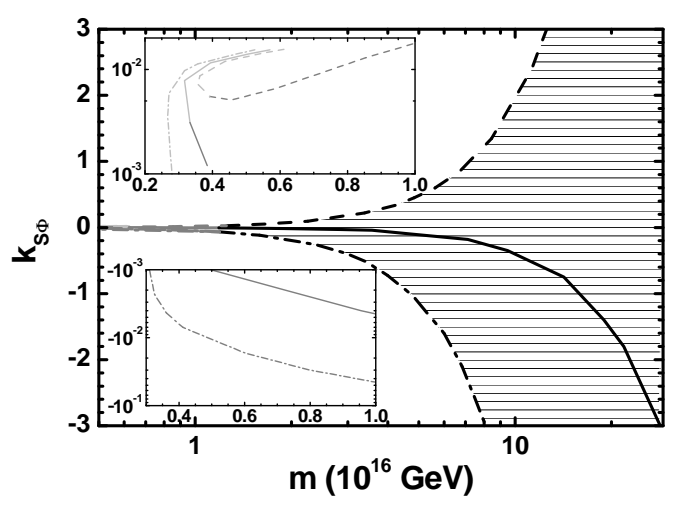

(b)

Figure 4: Allowed (hatched) region as determined by Eqs. (1.5), (1.7) and (1.9) in the $m-c_{R}\left[m-k_{S \Phi}\right]$ plane (a) [(b)] for $k_{S}=k_{\Phi}=0.5$. We follow the conventions of Fig. 1.

\section{Conclusions}

We reviewed the implementation of nMI in both a non-SUSY and a SUSY framework, by conveniently choosing the coupling function of the inflaton to gravity. In all cases the inflationary observables are compatible with the current data. In the SUSY cases the tachyonic instability, occurring along the direction of the accompanying non-inflaton field, can be remedied by considering terms up to the fourth order in the kinetic part of the frame function. Some of these terms assist in obtaining $n_{\mathrm{s}}$ close to its central observationally favored value. The role of the inflaton can be played by a gauge singlet or non-singlet superfield. In the latter case, though, a conjugation symmetry has to be imposed to the higher order terms so that the flatness of the potential is maintained and the radiative corrections turn out to be significant. For this reason, we can conclude that nMI within SUGRA is more naturally realized by a gauge singlet inflaton.

\section{References}

[1] C. Pallis, Phys. Lett. B 692, 287 (2010) [arXiv: 1002 . 4765].

[2] C. Pallis and N. Toumbas, JCAP 02, 019 (2011) [arXiv:1101.0325].

[3] C. Pallis and N. Toumbas, JCAP 12, 002 (2011) [arXiv:1108.1771];

C. Pallis and N. Toumbas, "Open Questions in Cosmology" (InTech, 2012) [arXiv: 1207.3730].

[4] C. Pallis and Q. Shafi, Phys. Rev. D 86, 023523 (2012) [arXiv: 1204 . 0252].

[5] P. Ade et al. [Planck Collaboration], arXiv:1303.5082.

[6] C.P. Burgess, H.M. Lee and M. Trott, JHEP 09, 103 (2009) [arXiv: 0902 . 4465].

[7] M.B. Einhorn and D.R.T. Jones, JHEP 03, 026 (2010) [arXiv: 0912 .2718];

H.M. Lee, JCAP 08, 003 (2010) [arXiv: 1005.2735];

S. Ferrara et al., Phys. Rev. D 83, 025008 (2011) [arXiv: 1008 .2942].

[8] R. Kallosh and A. Linde, JCAP 11, 011 (2010) [arXiv:1008.3375].

[9] J.L. Cervantes-Cota and H. Dehnen, Phys. Rev. D 51, 395 (1995) [astro-ph/9412032]; M. Arai, S. Kawai and N. Okada, Phys. Rev. D 84, 123515 (2011) [arXiv:1107.4767]; M.B. Einhorn and D.R.T. Jones, JCAP 11, 049 (2012) [arXiv:1207.1710]. 